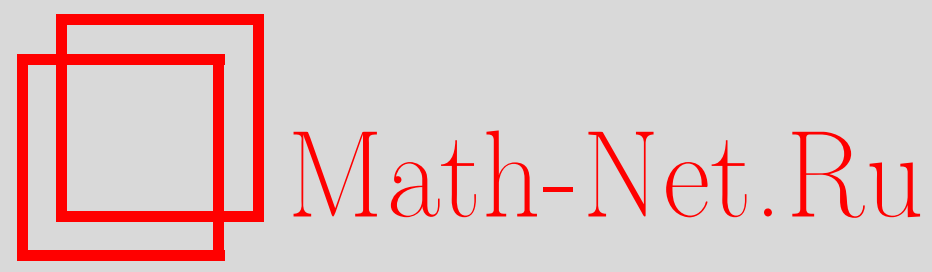

А. С. Мелузов, Построение эффективных алгоритмов решения систем полиномиальных булевых уравнений методом опробования части переменных, Дискрет. матем., 2011, том 23, выпуск 4, 66-79

DOI: https://doi.org/10.4213/dm1162

Использование Общероссийского математического портала Math-Net.Ru подразумевает, что вы прочитали и согласны с пользовательским соглашением http://www.mathnet.ru/rus/agreement

Параметры загрузки:

IP : 52.23 .180 .231

26 апреля 2023 г., $14: 20: 12$ 


\title{
Построение эффективных алгоритмов решения систем полиномиальных булевых уравнений методом опробования части переменных
}

\author{
(C) 2011 г. А. С. Мелузов
}

\begin{abstract}
В работе рассматривается комбинированный метод решения систем полиномиальных булевых уравнений, основанный на опробовании части переменных и отбрасывания части значений опробуемых переменных на основе критерия мономиальной совместимости. Рассчитана трудоемкость метода для некоторых значений параметров систем булевых уравнений.
\end{abstract}

\section{1. Введение}

В криптологии, теории кодирования и теории автоматов возникает задача изучения и решения систем булевых уравнений. Как известно, задача решения произвольной нелинейной системы полиномиальных уравнений является $N P$-трудной (см. [1]), и в настоящее время для решения таких систем не известно алгоритма, который бы имел сложность по порядку меньшую, чем $2^{c n}$, где $c-$ положительная постоянная, а $n-$ число булевых переменных в системе. В частности, сказанное выше справедливо и для квадратичных систем булевых уравнений. Поскольку в теории $N P$-полных задач сложность оценивается в худшем случае, то с теоретической и практической точки зрения актуальной является постановка задачи разработки эффективных алгоритмов решения для конкретных классов систем булевых уравнений.

В результате исследований были найдены и описаны классы систем уравнений, для которых существуют полиномиальные алгоритмы их решения (см., например, [2, 3]).

Универсальными алгебраическими методами решения полиномиальных систем булевых уравнений являются: построение с помощью алгоритма Бухбергера базиса Грёбнера идеала, описываемого системой уравнений (см. [4]); алгоритмы $F_{4}$ и $F_{5}$, разработанные Ж.-К. Фажере (см. [5, 6]); линеаризация системы с использованием дополнительных приемов повышения эффективности (см. [7, 8, 17, 18]). Для некоторых частных классов систем разработаны алгоритмы решения, использующие быстрый обход дерева решений системы (см. [9]), а также ассоциативные принципы использования памяти (см. [10]).

Настоящая работа посвящена построению и исследованию эффективности комбинированного метода решения систем нелинейных полиномиальных булевых уравнений, использующего опробование части переменных и критерий мономиальной совместности для отбраковки ложных вариантов значений опробованных переменных. 


\section{2. Постановка задачи}

Пусть $m, s$ - натуральные числа. Систему $m$ полиномиальных уравнений от $s$ переменных будем изображать в виде

$$
\begin{gathered}
g_{1}\left(x_{1}, x_{2}, \ldots, x_{s}\right)=a_{1}, \\
\cdots \\
g_{m}\left(x_{1}, x_{2}, \ldots, x_{s}\right)=a_{m},
\end{gathered}
$$

где $\left\{x_{1}, x_{2}, \ldots, x_{s}\right\}$ - множество булевых переменных, принимающих значения в $\mathbf{F}_{2}$, $a_{1}, a_{2}, \ldots, a_{s}$ - правые части, элементы поля $\mathbf{F}_{2},\left\{g_{1}, g_{2}, \ldots, g_{m}\right\}$ - полиномы с коэффициентами из поля $\mathbf{F}_{2}$ (без свободных членов), $d=\max _{i} \operatorname{deg}\left(g_{i}\right)$ - максимальная степень полинома системы.

Под опробованием переменной $x_{j}, 1 \leqslant j \leqslant s$, соответствующим значению $a \in \mathbf{F}_{2}$, мы будем понимать преобразование системы (1) в систему вида

$$
\begin{array}{r}
f_{1}\left(x_{1}, \ldots, x_{j-1}, x_{j+1}, \ldots, x_{s}\right)= \\
g_{1}\left(x_{1}, \ldots, x_{j-1}, a, x_{j+1}, \ldots, x_{s}\right) \\
\oplus g_{1}(0, \ldots, 0, a, 0, \ldots, 0)=a_{1}^{\prime} \\
\cdots \\
f_{m}\left(x_{1}, \ldots, x_{j-1}, x_{j+1}, \ldots, x_{s}\right)=g_{m}\left(x_{1}, \ldots, x_{j-1}, a, x_{j+1}, \ldots, x_{s}\right) \\
\oplus g_{m}(0, \ldots, 0, a, 0, \ldots, 0)=a_{m}^{\prime},
\end{array}
$$

где $\left\{f_{1}, \ldots, f_{m}\right\}$ - полиномы без свободных членов,

$$
a_{i}^{\prime}=a_{i} \oplus g_{i}(0, \ldots, 0, a, 0, \ldots, 0), \quad 1 \leqslant i \leqslant m .
$$

Аналогично определяется опробование нескольких переменных.

Пусть в (1) $a_{1}, \ldots, a_{m}, g_{1}, \ldots, g_{m}$ фиксированы. Требуется найти набор значений переменных $x_{1}, \ldots, x_{s}$, удовлетворяющий (1). Будем решать эту задачу, используя опробование части переменных. Аналогичный подход использовался в методе fix-XL (cм. гл. 12 в [11]) в одном из вариантов алгоритма XL-метода, в котором, перед применением самого алгоритма XL, часть переменных опробуется.

Выберем некоторое натуральное число $k, k<s$ и $n=s-k$. Без нарушения общности будем считать, что происходит опробование $k$ переменных $x_{n+1}, x_{n+2}, \ldots, x_{s}$. Очевидно, что степень полученных после преобразования полиномов $f_{1}, \ldots, f_{m}$ не превосходит $d$. Введем обозначение

$$
S_{n, d}=\sum_{i=1}^{d}\left(\begin{array}{l}
n \\
i
\end{array}\right) .
$$

Левую часть $i$-го уравнения системы (2) можно поставить во взаимно-однозначное соответствие с двоичным вектором длины $S_{n, d}$, который бы описывал наличие или отсутствие соответствующих мономов в полиномиальной записи $f_{i}$. Системы уравнений вида (2), получаемые с помощью опробования части переменных, будем называть редуцированными.

\section{3. Мономиальная совместность полиномиальных систем уравнений}

Нам потребуется еще одно понятие, которое будет использовано при построении критерия отбраковки ложных вариантов опробований. 
Определение 1. Система полиномиальных уравнений вида (1) называется мономиально совместной, если система линейных уравнений, полученная из исходной системы путем переобозначения всех мономов степени не ниже 1 символами новых переменных (этот процесс называется линеаризацией системы), является совместной относительно новых переменных. Наоборот, если получаемая после переобозначения система линейных уравнений несовместна относительно новых переменных, то исходная система называется мономиально несовместной.

Мономиальная совместность успешно используется в математическом аппарате исследований алгебраической иммунности, например, для определения наличия аннигиляторов степени не выше заданной (см. например, [12]).

Для иллюстрации понятия мономиальной совместности приведем следующие примеры.

Пример 1. Пример системы, которая является и мономиально совместной и совместной в обычном смысле:

$$
\begin{aligned}
a b & =1, \\
a+b & =0, \\
a b+a+b & =1 .
\end{aligned}
$$

Пример 2. Пример системы, которая является мономиально совместной, но не является совместной в обычном смысле:

$$
\begin{aligned}
a b & =1, \\
a+b & =1, \\
a b+a+b & =0 .
\end{aligned}
$$

Пример 3. Пример системы, которая является мономиально несовместной:

$$
\begin{aligned}
a b & =1, \\
a+b & =0, \\
a b+a+b & =0 .
\end{aligned}
$$

Предложение 1. Если система является мономиально несовместной, то она является несовместной и в обычном смысле.

Доказательство. Допустим, обратное. Пусть существует мономиально несовместная система уравнений, которая имеет решение в обычном смысле. Подставив значения соответствующих мономов в качестве значений переменных линеаризованной системы получим верное тождество, однако по условию, линеаризованная система решений не имеет. Получаем противоречие. Значит предположение неверно, что доказывает исходное утверждение.

Данное утверждение можно переформулировать следующим образом.

Следствие 1. Если система является совместной в обычном смысле, то она является мономиально совместной.

Совместность линейных систем уравнений может быть исследована с помощью одного из известных алгоритмов (например, алгоритм Гаусса решения систем линейных уравнений в качестве побочного результата определяет совместность системы) за время порядка $O\left(T^{\omega}\right)$, где $T$ - максимальный из размеров матрицы системы, а $\omega-$ коэффициент, который зависит от используемого алгоритма. В случае использования алгоритма Гаусса, $\omega=3$, а при использовании алгоритма Штрассена (см. [13]) $\omega=\log _{2} 7$. 


\section{4. Описание метода решения систем уравнений}

Предлагаемый метод (первый его этап) решения систем полиномиальных уравнений состоит в частичном опробовании переменных исходной системы и дальнейшей проверке полученной системы на мономиальную совместность. Приведем формальное описание метода решения системы (1) и оценим трудоемкость метода. За единичную операцию в приводимых выражениях трудоемкости примем операцию опробования переменных.

Не ограничивая общности рассуждений, будем опробовать значения переменных $\left\{x_{n+1}, \ldots, x_{s}\right\}$ для получения системы вида (2).

На первом этапе для каждого фиксированного набора значений $\left\{x_{n+1}, \ldots, x_{s}\right\}$ будем исследовать мономиальную совместность соответствующей редуцированной системы. В случае, если редуцированная система несовместна, то мы отбрасываем данный набор опробуемых значений как невозможный вариант части полного решения системы. Трудоемкость данного этапа можно оценить выражением $2^{k}\left(1+C_{\omega}(m, n, d)\right)$, где 1 - трудоемкость получения системы (2) из системы $(1)$, а $C_{\omega}(m, n, d)$ - трудоемкость проверки на совместность соответствующей линейной системы. Например, в случае, если для этого будет использоваться метод Гаусса, то $C_{\omega}(m, n, d)$ можно сверху асимптотически оценить как $O\left(\left(\max \left\{m, S_{n, d}\right\}\right)^{3}\right)$.

На втором этапе проведем проверку оставшихся вариантов путем решения редуцированных систем уравнений одним из указанных в разделе 1 методов, либо полным перебором оставшихся $n$ переменных. Тогда трудоемкость этого этапа не превосходит

$$
p 2^{k} 2^{c_{1} n}
$$

где $p$ - доля мономиально совместных систем среди всех, полученных при опробованиях, $c_{1}, 0<c_{1} \leqslant 1,-$ константа, зависящая от метода, который выбран для решения систем, не отброшенных на первом этапе. Константа $c_{1}$ равна 1 при использовании полного перебора.

Общая трудоемкость метода, таким образом, составит

$$
2^{k}\left(1+C_{\omega}(m, n, d)+p 2^{c_{1} n}\right) .
$$

Отметим, что надежность описанного метода равна единице, то есть при любой исходной системе (1) все ее решения будут найдены и любое найденное решение будет решением исходной системы, что следует из предложения 1 и следствия 1.

Приведем формализованное описание алгоритма, реализующего описанный метод решения систем полиномиальных булевых уравнений. На вход подается система уравнений (1) и набор опробуемых переменных $\left\{x_{n+1}, \ldots, x_{s}\right\}$. На выходе алгоритм выдает наборы значений переменных системы (1), являющиеся решениями.

\section{Алгоритм $A$.}

(1) Выбираем следующий по порядку набор значений для опробования переменных $\left\{x_{n+1}, \ldots, x_{s}\right\}$ и, подставляя выбранные значения, получаем редуцированную систему уравнений. Если все возможные значения опробуемых переменных исследованы, завершаем алгоритм.

(2) Выполняем линеаризацию полученной на предыдущем шаге редуцированной системы уравнений, получая линейную систему уравнений. 
(3) Проверяем полученную линейную систему уравнений на совместность, например, методом Гаусса. Если линейная система несовместна, переходим к шагу 1. В противном случае переходим к следующему шагу.

(4) Решаем редуцированную мономиально совместную систему, например, полным перебором (или используя результаты, полученные на шаге 3). Полученные решения совместно с текущими значениями опробуемых переменных составляют решение исходной системы - подаем их на выход алгоритма. Переходим к шагу 1.

\section{5. Расчет параметров метода}

\section{1. Рассматриваемое вероятностное пространство}

Трудоемкость метода (4), очевидно, зависит от самой системы, а именно, от $p$ - отношения числа мономиально совместных систем к общему числу систем, полученных при опробовании переменных. Будем оценивать $p=p(n, d, m)$ в следующем вероятностном пространстве.

Пусть $M_{s, d}$ - множество всех мономов степени не выше $d$ от $s$ переменных. Например, $M_{3,2}=\left\{1, x_{1}, x_{2}, x_{3}, x_{1} x_{2}, x_{1} x_{3}, x_{2} x_{3}\right\}$. Тогда любой полином степени не выше $d$ от $s$ переменных может однозначно задаваться вектором длины $\left|M_{s, d}\right|=S_{s, d}+1$ коэффициентов при соответствующих мономах из множества $M_{s, d}$. Тогда система полиномиальных булевых уравнений от $s$ переменных, степени не выше $d$, состоящая из $m$ уравнений однозначно определяется матрицей коэффициентов $K^{\left(S_{s, d}+1\right) \times m}$, в которой каждая строка представляет собой вектор коэффициентов при мономах, задающий соответствующее уравнение системы. Заметим, что всего различных матриц коэффициентов $2^{m\left(S_{s, d}+1\right)}$, однако различные матрицы могут задавать эквивалентные системы булевых уравнений.

Рассмотрим теперь следующее вероятностное пространство, зависящее от параметров $m, s$ и $d$. Множество элементарных исходов $\Omega$ состоит из всех возможных полиномиальных систем булевых уравнений, соответствующих всем возможным матрицам коэффициентов. Все элементарные исходы равновероятны.

Рассмотрим теперь набор случайных величин $\alpha_{i, j}, 0 \leqslant i \leqslant S_{s, d}, 1 \leqslant j \leqslant m$, представляющих собой коэффициенты при $i$-м мономе в $j$-м уравнении системы $\left(\alpha_{0, j}-\right.$ коэффициент при свободном члене $j$-го уравнения). Как несложно видеть, в данном вероятностном пространстве все случайные величины $\alpha_{i, j}$ являются независимыми в совокупности случайными величинами и принимают значения 0 и 1 с вероятностью $1 / 2$.

Будем рассматривать систему булевых полиномиальных уравнений со случайными коэффициентами при мономах

$$
\begin{aligned}
\alpha_{1,1} \mu_{1}+\alpha_{2,1} \mu_{2}+\ldots+\alpha_{S_{s, d}, 1} \mu_{S_{s, d}} & =\alpha_{0,1}, \\
& \ldots \\
\alpha_{1, m} \mu_{1}+\alpha_{2, m} \mu_{2}+\ldots+\alpha_{S_{s, d}, m} \mu_{S_{s, d}} & =\alpha_{0, m},
\end{aligned}
$$

где $\mu_{i}-i$-й моном множества $M, \alpha_{0, j}$ - коэффициент при мономе $\mu_{0}=1$ в $j$-м уравнении, то есть свободный член.

Пусть выбрано подмножество $X$ множества всех переменных и, без ограничения общности, пусть $X=\left\{x_{n+1}, \ldots, x_{s}\right\}$. Множество мономов $M$ может быть разбито на два непересекающихся множества $M_{X}$ и $M_{\bar{X}}$, где $M_{\bar{X}}$ состоит из всех элементов $M$, которые 
не содержат в себе переменных из $X$, а $M_{X}$ - из всех остальных мономов. Заметим, что моном 1 входит в $M_{\bar{X}}$ и что $\left|M_{\bar{X}}\right|=S_{n, d}+1$.

После опробования переменных из $X$ значениями вектора опробования $\tilde{a}$ получим из системы (5) систему следующего вида:

$$
\begin{gathered}
\beta_{1,1} v_{1}+\beta_{2,1} v_{2}+\ldots+\beta_{S_{n, d}, 1} v_{S_{n, d}}=\beta_{0,1}, \\
\cdots \\
\beta_{1, m} v_{1}+\beta_{2, m} v_{2}+\ldots+\beta_{S_{n, d}, m} v_{S_{n, d}}=\beta_{0, m},
\end{gathered}
$$

где $v_{i}-i$-й моном множества $M_{\bar{X}}, \beta_{i, j}$ - случайная величина, коэффициент при $i$-м мономе $j$-го уравнения, $\beta_{0, j}-$ коэффициент при свободном члене (мономе 1 ) в $j$-м уравнении.

Для коэффициентов при мономах в полученной системе верна следующая лемма.

Лемма 1. Все коэффициенты $\beta_{i, j}$ в системе (6), полученной из (5) в результате опробования переменных из $X$ значениями вектора $\tilde{a}$, являются независимыми в совокупности случайными величинами, принимающими значения 0 и 1 с вероятностями $p=1 / 2$.

Доказательство. Каждому моному $v_{i}, v_{i} \in M_{\bar{X}}$, поставим в соответствие такое множество $M_{X}^{i}, M_{X}^{i} \subset M_{X}$, которое содержит все мономы из $M_{X}$, которые при опробовании переменных из $X$ единицами становятся тождественно равны $v_{i}$. Обозначим $H_{i}$ множество индексов, которые соответствуют в системе (5) мономам из $M_{X}^{i}$. Кроме того, для всех номеров $h \in H_{i}$ для всех $i$ и каждого вектора опробования $\tilde{a}$ введем параметр $a_{h}$, который равен единице, если на векторе опробования $\tilde{a}$ моном $\mu_{h}$ становится тождественно равен моному $v_{i}$, и равный нулю, если на векторе опробования $\tilde{a}$ моном $\mu_{h}$ тождественно равен 0 .

Пусть кроме того, каждому индексу $i$ монома $v_{i} \in M_{\bar{X}}$ соответствует индекс $r_{i}$ такой, что $v_{i} \equiv \mu_{r_{i}}$, где $\mu_{r_{i}} \in M$, то есть моном с индексом $i$ в системе (6) совпадает с мономом с индексом $r_{i}$ в системе (5). Данное соответствие является взаимно-однозначным.

Тогда несложно видеть, что для любой пары $i, j, 0 \leqslant i \leqslant S_{n, d}, 1 \leqslant j \leqslant m$, справедливо равенство

$$
\beta_{i, j}=\alpha_{r_{i}, j} \oplus \bigoplus_{h \in H_{i}} a_{h} \alpha_{h, j}
$$

Заметим, что случайные величины $\alpha_{r_{i}, j}$ и $\delta_{i, j}=\bigoplus_{h \in H_{i}} a_{h} \cdot \alpha_{h, j}$ независимы, поскольку $r_{i} \notin H_{i}$.

Пусть сумма $\bigoplus_{h \in H_{i}} a_{h} \alpha_{h, j}$ принимает значение 1 с вероятностью $p_{i, j}$ и значение 0 с вероятностью $\left(1-p_{i, j}\right)$. Тогда, поскольку $\alpha_{r_{i}, j}$ принимает значения 0 и 1 с вероятностями $1 / 2$, случайная величина $\beta_{i, j}$ принимает значение 0 с вероятностью

$$
\mathbf{P}\left(\alpha_{r_{i}, j}=0\right) \mathbf{P}\left(\delta_{i, j}=0\right)+\mathbf{P}\left(\alpha_{r_{i}, j}=1\right) \mathbf{P}\left(\delta_{i, j}=1\right)=\frac{1}{2} p_{i, j}+\frac{1}{2}\left(1-p_{i, j}\right)=\frac{1}{2} .
$$

Аналогично, $\beta_{i, j}$ принимает значение 1 с вероятностью $1 / 2$.

Поскольку случайные величины $\alpha_{i, j}$ являются независимыми в совокупности, то соответствующие случайные величины $\beta_{i, j}$ также являются независимыми в совокупности.

Таким образом, если получаемые после опробования редуцированные системы полиномиальных уравнений линеаризовать, то расширенная матрица коэффициентов линеаризованной системы $[A \mid b]$ состоит из независимых в совокупности случайных величин, 
принимающих значения 0 и 1 с вероятностью 1/2 (здесь $A$ - матрица коэффициентов при мономах степени не ниже 1 в левой части, а $b-$ вектор-столбец правых частей, коэффициенты при константах). Заметим, что сходная идея использования вероятностной модели применена в [14] для исследования комбинирующего генератора.

\section{2. Оценка ранга системы линейных уравнений}

В соответствии с критерием Кронекера-Капелли, система линейных уравнений совместна тогда и только тогда, когда ранг матрицы коэффициентов системы равен рангу расширенной матрицы коэффициентов системы $(\operatorname{rank}(A)=\operatorname{rank}(A \mid b))$. Этот же критерий, в соответствии с определением 1 , можно использовать и для определения мономиальной совместности систем полиномиальных уравнений.

Следующие предельный и точный результаты позволяют вычислять вероятность принятия рангом случайной системы конкретного значения.

Предложение 2 ([15, теорема 3.2.1]). Пусть матрица A имеет размеры $m \times T$, ее элементы - независимые случайные величины, принимающие значения 0 и 1 с вероятностью 1/2. Обозначим $\rho(m)$ случайную величину, соответствующую рангу матриць $A$.

Пусть $q \geqslant 0$ и $t-$ фиксированные челье числа, $t+q \geqslant 0$. Тогда, если $T \rightarrow \infty u$ $m=T+t$, mo

$$
\mathbf{P}\{\rho(m)=T-q\} \rightarrow 2^{-q(t+q)} \prod_{i=q+1}^{\infty}\left(1-2^{-i}\right) \prod_{i=1}^{t+q}\left(1-2^{-i}\right)^{-1},
$$

где последнее произведение полагается равным единице при $t+q=0$.

Предложение 3 ([16], соотношение (2.4)). Пусть матрица $A$ имеет размеры $m \times T$, ее элементы - независимые случайные величины, принимающие значения 0 и 1 с вероятностью $1 / 2$. Пусть $\rho(m)-$ случайная величина, соответствующую рангу матрицы А.

Пусть $q \geqslant 0$ и $t$ - фиксированные целье числа, $t+q \geqslant 0, m=T+t$. Тогда $\rho(m)$ имеет следующее распределение:

$$
\mathbf{P}\{\rho(m)=T-q\}=2^{-q(t+q)} \frac{\prod_{i=q+1}^{T}\left(1-2^{-i}\right) \prod_{i=q+t+1}^{T+t}\left(1-2^{-i}\right)}{\prod_{i=1}^{T-q}\left(1-2^{-i}\right)} .
$$

Здесь и далее принимаем произведение $\prod_{i=u}^{v}\left(1-2^{-i}\right)$ равным 1 при $u>v$.

В дальнейшем мы будем использовать для получения оценок трудоемкости рассматриваемого метода только результат (3).

\section{3. Оценка вероятности совместности системы линейных уравнений}

Рассмотрим систему линейных уравнений, порожденную матрицей $(A \mid b)$. Пусть $m \geqslant T$. Тогда верно следующее утверждение.

Лемма 2. Для вероятности совместности системь линейных уравнений, порожденной матрицей $(A \mid b)$ справедливо следующее соотношение:

$$
\begin{aligned}
& \mathbf{P}[\operatorname{rank}(A)=\operatorname{rank}(A \mid b)] \\
& \quad=\sum_{i=0}^{T}\left(2^{-(T-i+1)(m-i)} \frac{\prod_{j=T-i+1}^{T}\left(1-2^{-j}\right) \prod_{j=m-i+1}^{m}\left(1-2^{-j}\right)}{\prod_{j=1}^{i}\left(1-2^{-j}\right)}\right) .
\end{aligned}
$$


Доказательство. Рассмотрим матрицу $A$. Ее ранг может принимать различные значения с вероятностями, определяемыми соотношением (3).

Допустим, матрица $A$ имеет ранг $i$. Это означает, что в $A$ можно выделить $i$ линейно независимых главных строк $A_{k_{1}}, A_{k_{2}}, \ldots, A_{k_{i}}$, а строка $A_{j}$ матрицы $A$ может единственным способом быть представлена как линейная комбинация

$$
A_{j}=c_{j_{1}} A_{k_{1}}+c_{j_{2}} A_{k_{2}}+\ldots+c_{j_{i}} A_{k_{i}},
$$

где $c_{j_{1}}, \ldots, c_{j_{i}}$ - константы 0 и 1 . Обозначим $A b_{j} j$-ю строку расширенной матрицы $[A \mid b]$. Поскольку строки $A_{k_{1}}, A_{k_{2}}, \ldots, A_{k_{i}}$ линейно независимы и являются подстроками $A b_{k_{1}}, A b_{k_{2}}, \ldots, A b_{k_{i}}$, значит строки $A b_{k_{1}}, A b_{k_{2}}, \ldots, A b_{k_{i}}$ также являются линейно независимыми. Для того, чтобы ранг расширенной матрицы $[A \mid b]$ совпадал с рангом $A$ необходимо, чтобы для любого номера строки $j$ выполнялось равенство

$$
A b_{j}=c_{j_{1}} A b_{k_{1}}+c_{j_{2}} A b_{k_{2}}+\ldots+c_{j_{i}} A b_{k_{i}} .
$$

Следовательно, для равенства рангов $A$ и $[A \mid b]$ необходимо, чтобы для любого $j$, $1 \leqslant j \leqslant m$

$$
b_{j}=c_{j_{1}} b_{k_{1}}+c_{j_{2}} b_{k_{2}}+\ldots+c_{j_{i}} b_{k_{i}} .
$$

Поскольку случайные величины, входящие в вектор $b$, в описанном вероятностном пространстве независимы и принимают значения 0 и 1 с равными вероятностями, вероятность выполнения условия (11) равна $1 / 2$.

Значит, если ранг матрицы $A$ равен $i$, вероятность того, что ранг матрицы $A \mid b$ также равен $i$, равна

$$
\mathbf{P}[\operatorname{rank}(A \mid b)=i \mid \operatorname{rank}(A)=i]=\frac{1}{2^{m-i}} .
$$

Далее, по формуле условной вероятности получаем, что

$$
\begin{aligned}
\mathbf{P}[\operatorname{rank}(A)=\operatorname{rank}(A \mid b)] & =\sum_{i=0}^{T} \mathbf{P}[\operatorname{rank}(A)=\operatorname{rank}(A \mid b)=i] \\
& =\sum_{i=0}^{T}(\mathbf{P}[\operatorname{rank}(A)=i] \mathbf{P}[\operatorname{rank}(A \mid b)=i \mid \operatorname{rank}(A)=i]) \\
& =\sum_{i=0}^{T}\left(\mathbf{P}[\operatorname{rank} A=i] \frac{1}{2^{m-i}}\right) .
\end{aligned}
$$

Подставив сюда выражение (3), получим, что

$$
\begin{aligned}
& \mathbf{P}[\operatorname{rank}(A)=\operatorname{rank}(A \mid b)] \\
& =\sum_{i=0}^{T}\left(\frac{1}{2^{m-i}} \frac{1}{2^{(T-i)(m-T+T-i)}} \frac{\prod_{j=T-i+1}^{T}\left(1-2^{-j}\right) \prod_{j=m-i+1}^{m}\left(1-2^{-j}\right)}{\prod_{j=1}^{i}\left(1-2^{-j}\right)}\right) \\
& =\sum_{i=0}^{T}\left(2^{-(T-i+1)(m-i)} \frac{\prod_{j=T-i+1}^{T}\left(1-2^{-j}\right) \prod_{j=m-i+1}^{m}\left(1-2^{-j}\right)}{\prod_{j=1}^{i}\left(1-2^{-j}\right)}\right) .
\end{aligned}
$$

Обозначим правую часть выражения (10) через $\mathscr{P}(T, m)$. 


\section{4. Зависимость вероятности совместности системы линейных уравнений от параметров системы}

Исследуем поведение $\mathscr{P}(T, m)$ при заданной разности между $m$ и $T$.

Пусть $m=T+l$, тогда обозначим $\mathscr{P}(T, T+l)$ через $\mathscr{P}_{T}(l)$.

Докажем теперь следующее утверждение, характеризующее зависимость $\mathscr{P}_{T}(l)$ от разности $l$ между сторонами матрицы.

Лемма 3. Для любых $z \geqslant 0, l \geqslant 1, T \geqslant 1$

$$
\mathscr{P}_{T}(l+z) \leqslant \frac{1}{2^{z-2}} \mathscr{P}_{T}(l) .
$$

Доказательство. Заметим, что переобозначив в выражении для $\mathscr{P}(T, T+l)$ индекс суммирования $i^{\prime}=T-i+1$, получим следующее выражение

$$
\begin{aligned}
\mathscr{P}(T, T+l) & =\sum_{i=0}^{T}\left(2^{-(T-i+1)(T+l-i)} \frac{\prod_{j=T-i+1}^{T}\left(1-2^{-j}\right) \prod_{j=T+l-i+1}^{T+l}\left(1-2^{-j}\right)}{\prod_{j=1}^{i}\left(1-2^{-j}\right)}\right) \\
& =\sum_{i=1}^{T+1}\left(\frac{1}{2^{i(l+i-1)}} \frac{\prod_{j=i}^{T}\left(1-2^{-j}\right) \prod_{j=i+l}^{T+l}\left(1-2^{-j}\right)}{\prod_{j=1}^{T-i+1}\left(1-2^{-j}\right)}\right)=\mathscr{P}_{T}(l) .
\end{aligned}
$$

В соответствии с полученным выражением, выпишем формулу для $\mathscr{P}_{T}(l+z)$ :

$$
\begin{aligned}
\mathscr{P}_{T}(l+z) & =\sum_{i=1}^{T+1} \frac{1}{2^{i(l+z+i-1)}} \frac{\prod_{j=i}^{T}\left(1-2^{-j}\right) \prod_{j=i+l+z}^{T+l+z}\left(1-2^{-j}\right.}{\prod_{j=1}^{T-i+1}\left(1-2^{-j}\right)} \\
& =\sum_{i=1}^{T+1} \frac{1}{2^{i(l+i-1)}} \frac{1}{2^{i z}} \frac{\prod_{j=i}^{T}\left(1-2^{-j}\right) \prod_{j=i+l}^{T+l}\left(1-2^{-j}\right)}{\prod_{j=1}^{T-i+1}\left(1-2^{-j}\right)} \frac{\prod_{j=T+l+1}^{T+l+z}\left(1-2^{-j}\right)}{\prod_{j=i+l}^{i+l+z-1}\left(1-2^{-j}\right)} .
\end{aligned}
$$

Очевидно, что для любого $i \geqslant 1$

$$
\frac{1}{2^{i z}} \leqslant \frac{1}{2^{z}}
$$

Кроме того, в условиях леммы

$$
\frac{\prod_{j=T+l+1}^{T+l+z}\left(1-2^{-j}\right)}{\prod_{j=i+l}^{i+l+z-1}\left(1-2^{-j}\right)}<4
$$

Действительно, известно, что $\prod_{n=1}^{\infty}\left(1-2^{-n}\right) \approx 0,2887880950866024 \ldots$, поэтому для для всех $i \geqslant 1, z \geqslant 0, l \geqslant 1$

$$
\prod_{j=i+l}^{i+l+z-1}\left(1-2^{-j}\right)>0,288788, \quad \prod_{j=T+l+1}^{T+l+z}\left(1-2^{-j}\right)<1 .
$$

Значит,

$$
\mathscr{P}_{T}(l+z) \leqslant \sum_{i=1}^{T+1} \frac{1}{2^{i(l+i-1)}} \frac{1}{2^{z-2}} \frac{\prod_{j=i}^{T}\left(1-2^{-j}\right) \prod_{j=i+l}^{T+l}\left(1-2^{-j}\right)}{\prod_{j=1}^{T-i+1}\left(1-2^{-j}\right)}=\mathscr{P}_{T}(l) \frac{1}{2^{z-2}} .
$$




\section{5. Оценка трудоемкости метода решения систем полиномиальных уравнений}

Заметим, что для случайных величин $\beta_{i, j}$, соответствующих коэффициентам при мономах в системе (6), для любого множества опробуемых переменных $X$ и любого вектора опробования $\tilde{a}$ выполняются условия предложения 3. Сформулируем и докажем следующую теорему.

Теорема 1. Пусть, как это описано выше, задано множество элементарных исходов $\Omega$ - множество систем вида (5) из т полиномиальных уравнений степени не выле $d$ над полем GF(2) от s неизвестных. Пусть $n$ - наибольшее среди иельх положительных решений неравенства

$$
m-S_{n, d}>n+2 .
$$

Пусть $\gamma$ - случайная величина, равная трудоемкости решения системь уравнений алгоритмом $A$, описанным в разделе 4, при опробовании $k=s-n$ переменных (заданных множеством $X,|X|=k)$, в предположении независимости и равномерного распределения коэффициентов системы (5). Тогда математическое ожидание $\mathbf{E}(\gamma)$ имеет верхнюю асимптотическую оценку $O\left(2^{k} \mathrm{~m}^{3}\right)$.

Доказательство. Обозначим $\gamma_{X, \tilde{a}}$ случайную величину, равную трудоемкости проверки на мономиальную совместность и, в случае мономиальной совместности, дальнейшего решения системы уравнений, полученной из выбранной случайно из множества $\Omega$ опробованием переменных из множества $X$ вектором $\tilde{a}$. Тогда, общая трудоемкость $\gamma$ может быть представлена в виде суммы

$$
\gamma=\sum_{\tilde{a} \in \mathbf{B}_{k}} \gamma_{X, \tilde{a}},
$$

где $\mathbf{B}_{k}-$ множество всех двоичных векторов длины $k$. Следовательно, по свойству математического ожидания,

$$
\mathbf{E}(\gamma)=\sum_{\tilde{a} \in \mathbf{B}_{k}} \mathbf{E}\left(\gamma_{X, \tilde{a}}\right) .
$$

Рассмотрим теперь случайную величину $\gamma_{X, \tilde{a}}$ для некоторого $\tilde{a}$. Значение $\gamma_{X, \tilde{a}}$ складывается из трудоемкости линеаризации и трудоемкости решения системы, в случае ее мономиальной совместности. При линеаризации системы уравнений (6), полученной после опробования $k=(s-n)$ переменных, число переменных $T$ линеаризованной системы равно

$$
S_{n, d}=\sum_{i=1}^{d}\left(\begin{array}{l}
n \\
i
\end{array}\right) .
$$

Трудоемкость решения редуцированной системы $\gamma_{X, \tilde{a}}$ равна $1+C_{\omega}(m, n, d)+I_{p} \cdot 2^{c_{1} n}$, где $C_{\omega}(m, n, d)$ - трудоемкость линеаризации, $I_{p}-$ индикаторная случайная величина, принимающая значение 1 с вероятностью $p$ и значение 0 с вероятностью $(1-p)$, где $p$ - вероятность мономиальной совместности редуцированной системы. Математическое ожидание $I_{p}$ равно, очевидно, $p$. На основании леммы 1 можно применять к линеаризованной системе утверждение 3 и лемму 2. В таком случае, $p$ можно оценивать сверху значением $\mathscr{P}_{T}(l)$ (см. (10)) 
Поскольку, по условию теоремы,

$$
l=m-T=m-S_{n, d}>n+2,
$$

значит, для оценки вероятности совместности получаемой при опробовании редуцированной системы $\mathscr{P}_{T}(l)$ может быть применен результат леммы 3 , а следовательно, математическое ожидание $\gamma_{X, \tilde{a}}$ при любом $\tilde{a}$ можно оценить как

$$
\begin{aligned}
\mathscr{P}_{T}(l) 2^{c_{1} n}+C_{\omega}(m, n, d) & <\mathscr{P}_{T}(0) \frac{1}{2^{l-2}} 2^{c_{1} n}+C_{\omega}(m, n, d) \\
& <\mathscr{P}_{T}(0) \frac{1}{2^{n}} 2^{c_{1} n}+C_{\omega}(m, n, d) .
\end{aligned}
$$

Поскольку $C_{\omega}(m, n, d)$ - трудоемкость решения системы линейных уравнений с матрицей размера $T \times m$ асимптотически сверху ограничена, другими словами есть $O\left(m^{3}\right)$ и $c_{1} \leqslant 1$ в соответствии с изложенным в разделе 4 , а $\mathscr{P}_{T}(0)<1$, значит, верхняя асимптотическая оценка математического ожидания $\gamma_{X, \tilde{a}}$ есть $O\left(m^{3}\right)$ для любого $\tilde{a}$.

Отсюда, на основании (15), получаем, что

$$
\mathbf{E}(\gamma)=\sum_{\tilde{a} \in \mathbf{B}_{k}} \mathbf{E}\left(\gamma_{X, \tilde{a}}\right)=\sum_{i=0}^{2^{k}-1} O\left(m^{3}\right)=O\left(2^{k} m^{3}\right) .
$$

\section{6. Оценка трудоемкости решения системы квадратичных уравнений}

Особого внимания заслуживает класс систем полиномиальных булевых уравнений, степень которых не превосходит 2, поскольку система уравнений любой степени может быть преобразована в квадратичную. Кроме того, в 2002 г. Н. Куртуа и Й. Пепржек [18] показали возможность сведения криптоанализа алгоритмов Rijndael и Serpent к решению системы булевых полиномиальных уравнений степени 2.

Для случая квадратичных систем уравнений можно получить следующее следствие из доказанной выше теоремы.

Следствие 2. Пусть множество элементарных исходов $\Omega-$ множество систем вида (5) из $m$ полиномиальных уравнений степени не выше 2 над полем GF(2) от s неизвестных. Пусть $n$ - наибольшее среди цельх положительных решений неравенства

$$
m-S_{n, d}>n+2 \text {. }
$$

Пусть $\gamma$ - случайная величина, равная трудоемкости решения системы уравнений алгоритмом $A$, описанным в разделе 4 , при опробовании $k=s-n$ переменных (заданных множеством $X,|X|=k)$, в предположении независимости и равномерного распределения коэффициентов системы (5). Тогда математическое ожидание

$$
\mathbf{E}(\gamma)=O\left(2^{(s-(\sqrt{8 m-7}-3) / 2} m^{3}\right) .
$$

Доказательство. Подставив $d=2$ в неравенство из условия теоремы 1 , получим квадратное неравенство $n^{2}+3 n-2 m+4<0$. Максимальное целочисленное $n$, удовлетворяющее неравенству может быть выражено как $\lfloor(\sqrt{8 m-7}-3) / 2\rfloor$. Отсюда, выбрав полученное решение в качестве $n$ в условиях теоремы 1 , получим асимптотическую оценку сверху $O\left(2^{(s-(\sqrt{8 m-7}-3) / 2} m^{3}\right)$. Следствие доказано. 
Аналогичные оценки могут быть получены и для систем алгебраических уравнений любой степени $d$, что потребует аналитического решения соответствующих неравенств степени $d$.

\section{6. Заключение}

В криптоанализе часто возникают переопределенные системы уравнений, заведомо имеющие заранее известное небольшое число решений $r$, причем, наиболее распространен случай, когда $r=1$. Несмотря на то, что такие системы неслучайны, однако, с точки зрения мономиальной структуры они достаточно разнообразны и к ним могут быть применены подходы, описываемые в настоящей статье.

Предложим следующую модификацию алгоритма $A$, которая представляется более эффективной при решении полиномиальных уравнений с известным числом решений определенного вида.

В случае сильно переопределенной системы квадратичных уравнений степени не выше 2 над полем $G F(2)$ от $s$ переменных, когда число уравнений $m$ превосходит $\left(s^{2}+3 s+4\right) / 2$, а число решений $r$, значительно большее $2^{s}$, известно заранее, можно предложить следующий подход к решению системы.

Будем опробовать по $k=\left\lceil\log _{2}(r+1)\right\rceil$ переменных. Опробуем сначала первые $k$ переменных $x_{1}, \ldots, x_{k}$. Для каждого вектора опробования получим редуцированную систему уравнений.

Проверим полученные системы на мономиальную совместность. В случае мономиальной совместности системы, проводим опробование следующих $k$ переменных, получая новые системы уравнений уже от $s-2 k$ переменных. Таким образом, проводя опробование в глубину мы получим дерево систем уравнений, на листьях которого будут решения исходной системы. Такой подход в некоторых случаях может быть более эффективен, чем алгоритм $A$.

Необходимо отметить, что полученные в разделе 5 оценки трудоемкости верны для случая, когда в качестве алгоритма решения редуцированных систем используется полный перебор. При использовании в качестве алгоритмов решения систем булевых уравнений иных (менее трудоемких) методов, упомянутых во введении, можно получить снижение оценок трудоемкости. Полученная в лемме 3 скорость убывания вероятности мономиальной совместности системы булевых уравнений может быть уточнена в сторону уменьшения коэффициента. Кроме того, возможно выделять классы булевых систем уравнений, для которых данный коэффициент будет еще меньше.

Применяя к исходной системе (1) метод линеаризации, будем получать линейную систему, имеющую $2^{S_{s, d}-w}$ решений, требующих проверки. В данном случае $w$ - ранг линеаризованной системы. В случае, если для исходной системы верно, что $m$ значительно меньше, чем $S_{s, d}$, будем получать $w \approx m$ и тогда трудоемкость метода линеаризации можно асимптотически оценить как $O\left(2^{S_{s, d}-m} C_{\text {test }}\right)$, где $C_{\text {test }}-$ трудоемкость проверки одного решения линеаризованной системы. Даже в случае $d=2$ получим $O\left(2^{\left(s^{2}+s\right) / 2-m} C_{\text {test }}\right)$. При тех же условиях, приведенный в данной статье метод будет иметь асимптотику трудоемкости $O\left(2^{(s-\lfloor\sqrt{8 m-7}-3) / 2\rfloor} m^{3}\right)$. Несложно видеть, что метод прямой линеаризации проигрывает в трудоемкости за исключением случаев, когда $m \gtrsim s^{2}$. Однако, при $m \gtrsim s d$ можно применить описанную в текущем разделе модификацию метода решения систем с каскадным опробованием и получить оценки трудоемкости, близкие к оценкам трудоемкости метода линеаризации при небольшом количестве решений исходной системы. 
При использовании для решения исходного уравнения методик, предложенных в [9], заметное снижение трудоемкости наблюдается при решении систем, в которых каждое отдельное уравнение зависит не более чем от $t$, существенно меньшем, чем $s$, переменных. В то же время, предложенный в настоящей статье метод не накладывает подобных серьёзных ограничений на решаемые системы, поэтому сравнивать трудоемкости данных методов некорректно.

При использовании для решения системы булевых уравнений построения базиса Грёбнера идеала, описываемого системой уравнений, трудоемкость характеризуется трудоемкостью построения базиса Грёбнера. Наиболее эффективным алгоритмом построения базиса Грёбнера в настоящий момент является $F_{5}$ (см. [6]). Теоретические оценки трудоемкости для некоторых видов систем булевых полиномиальных уравнений приведены в [19], из которой следует, что трудоемкость алгоритма характеризуется размерами матриц, операции над которыми проводятся в ходе построения базиса Грёбнера. Размеры данных матриц определяются как $\left(\begin{array}{c}s \\ D_{\mathrm{reg}}\end{array}\right)$, где $D_{\text {reg }}-$ степень полурегулярности системы, определяемая как номер первого отрицательного коэффициента при разложении в ряд функции $(1-y)^{s} / \prod_{i=1}^{m}\left(1-y^{d_{i}}\right)$, где $d_{i}-$ степень $i$-го уравнения исходной системы. В общем случае, трудоемкость алгоритма будет превосходить трудоемкость полного перебора.

Предлагаемый в [17] XL-метод позволяет решать системы полиномиальных уравнений, состоящие из недостаточного для эффективного применения метода линеаризации числа уравнений. Для этого, путем домножения на всевозможные мономы ограниченной степени, повышается степень исходной системы и получаются вспомогательные уравнения, не несущие дополнительной информации в полиномиальной системе, однако позволяющие уточнить решения, получаемые при линеаризации. Данный метод эффективен при небольшом количестве мономов в уравнениях исходной системы, в то время как предложенный в данной статье метод не накладывает подобных ограничений. В отличие от XL-метода, где исходная система расширяется до тех пор, пока параметры системы не будут благоприятны для дальнейшей линеаризации, в предлагаемом в данной статье методе предполагается работа только с исходной системой и ее редукциями. Для XL-метода отсутствуют строго доказанные оценки его средней трудоемкости для конкретных классов систем уравнений. Поэтому сравнение эффективности XL-метода и рассмотренного в данной статье метода представляется затруднительным. Еще в большей степени это относится к сравнению предлагаемого метода и XSL-метода (см. [18]).

\section{Список литературы}

1. Гэри М., Джонсон Д., Вычислительные машины и труднорешаемые задачи. Мир, Москва, 1982.

2. Горшков С. П., Применение теории $N P$-полных задач для оценки сложности решения систем булевых уравнений. Обозрение прикладной и промышленной математики (1995) 2, №3, 325398.

3. Смирнов В. Г., Некоторые классы классы эффективно решаемых систем булевых уравнений. Труды по дискретной математике (2000) 3, 269-282.

4. О’Ши Д., Кокс Д., Литтл Д., Идеаль,, многообразия и алгоритмыл. Мир, Москва, 2000.

5. Faugère J.-C., A new efficient algorithm for computation Gröebner bases $\left(F_{4}\right)$. J. Pure Appl. Algebra (1999) 139, 61-88.

6. Faugère J.-C., A new efficient algorithm for computation Gröebner bases without reduction to zero $\left(F_{5}\right)$. In: Proc. ESSAC'2002 (Mora T., ed.). ACM Press, New York, 2002, pp. 75-83.

7. Courtois N., Meier W., Algebraic attacks on stream ciphers with linear feedback. Lecture Notes Computer Sci. (2003) 2656, 345-359. 
8. Meier W., Pasalic E., Carlet C., Algebraic attacks and decomposition of Boolean functions. Lecture Notes Computer Sci. (2004) 3027, 474-491.

9. Semaev I., On solving sparse algebraic equations over finite fields. Des. Codes Cryptography (2008) 49, 47-60.

10. Мелузов А. С., Использование ассоциативных принципов обработки информации для построения алгоритмов решения систем булевых уравнений. Журнал вычислительной математики и математической физики (2010) 50, №11, 2028-2044.

11. Bard G., Algebraic cryptanalysis. Springer, New York, 2009.

12. Лобанов М. С., Точное соотношение между нелинейностью и алгебраической иммунностью. Дискретная математика (2006) 18, №3, 152-159.

13. Strassen V., Gaussian elimination is not optimal. Numer. Math. (1969) 13, 354-356.

14. Логачев О. А., Сальников А. А., Ященко В. В., Корреляционная иммунность и реальная секретность. В сб.: Математика и безопасность информационных технологий. МЦНМО, Москва, 2004, c. 176-178.

15. Колчин В. Ф., Случайные графы. Наука, Москва, 2004.

16. Сачков В. Н., Системы случайных уравнений над конечными полями. Труды по дискретной математике (2004) 8, 176-178.

17. Courtois N., Klimov A., Patarin J., Shamir A., Efficient algorithms for solving overdefined systems of multivariate polynomial equations. Lecture Notes Computer Sci. (2000) 1807, 392-407.

18. Courtois N., Pieprzyk J., Cryptanalysis of block ciphers with overdefined systems of equations. Lecture Notes Computer Sci. (2002) 2501, 267-287.

19. Bardet M. T., Faugère J.-C., Salvy B., Complexity of Gröbner basis computation for semi-regular overdetermined sequences over GF(2) with solutions in GF(2), INRIA Research Report 5049, 2003.

Статья поступила 3.03.2011. 